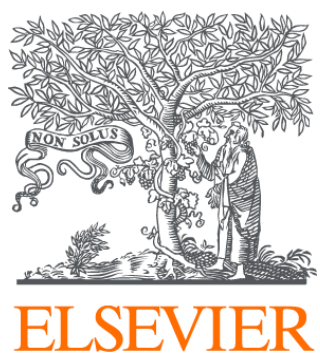

Since January 2020 Elsevier has created a COVID-19 resource centre with free information in English and Mandarin on the novel coronavirus COVID-

19. The COVID-19 resource centre is hosted on Elsevier Connect, the company's public news and information website.

Elsevier hereby grants permission to make all its COVID-19-related research that is available on the COVID-19 resource centre - including this research content - immediately available in PubMed Central and other publicly funded repositories, such as the WHO COVID database with rights for unrestricted research re-use and analyses in any form or by any means with acknowledgement of the original source. These permissions are granted for free by Elsevier for as long as the COVID-19 resource centre remains active. 


\section{Fever in the returning traveller}

\author{
Harriet Davidson \\ Angela Houston
}

\begin{abstract}
Global travel is increasingly a fact of modern life, and the rapid spread of severe acute respiratory syndrome coronavirus 2 leading to lockdown across the world has demonstrated the interconnectedness of the world's population. Illness in the returning traveller can range from trivial to life-threatening, and the concept of imported infection can be an intimidating diagnostic and management challenge. An important caveat is that even if your patient has returned from cuddling multimammate rats in Guinea 1 week ago, they could be febrile from a distinctly non-tropical urinary tract infection. That said, antimicrobial resistance is an established concern among returned travellers, which has further infection control implications. Infection control issues regarding isolation, personal protective equipment and notification to public health should always be considered for returning travellers on presentation often before diagnostic confirmation has been made. Always consider the risk of high-consequence infectious diseases.
\end{abstract}

Keywords Fever; imported fever; infection control; returning traveller; travel

\section{Introduction}

In 2019, UK residents made 93.1 million visits overseas, and overseas residents made 40.9 million visits to the UK. ${ }^{1}$ Before severe acute respiratory syndrome coronavirus 2, we could reasonably have expected this to increase year on year. A recent review found that $43-79 \%$ of returning travellers from developing countries become ill on return and may seek medical attention. $^{2}$ Fever in these travellers is caused by tropical infections in $36 \%$ of cases (malaria being the most common at $22 \%$ of febrile presentations) and non-tropical in 36\% (gastrointestinal infections, respiratory tract infections). ${ }^{3}$ This article aims to provide a framework to evaluate these patients. It is important to consider when the patient (who) has been doing what, where. The timing, exposures and location can often be usefully combined to prioritize testing. Working out the patient's clinical syndrome can also help guide a diagnosis.

\section{Harriet Davidson MA MRCP DTM\&H is a Registrar in Infectious Diseases and Microbiology at St George's Hospital NHS Foundation Trust, London, UK. Competing interests: none declared.}

Angela Houston FRCP FRCPath DTM\&H is a Consultant in Infectious Diseases and Microbiology at St George's Hospital NHS Foundation Trust, London, UK. Competing interests: none declared.

\section{Key points}

A thorough history and examination guide investigation and management

Malaria is the most common cause of febrile tropical infection

- always send a blood smear

Consider imported antimicrobial resistance

If in doubt, isolate and talk to the infectious disease team

\section{History}

The history taken from a returning traveller establishes the solid backbone of any clinical assessment. Without adequate assessment of the patient's travel destinations, exposures and timeline, it would be easy to inappropriately rule out, or not even consider, the true diagnosis. This can be broken down into four main categories: Who? Where? What? When?

\section{Personal factors: the 'Who?'}

Consider the patient and their personal risk factors for disease acquisition abroad. A personal medical history and drug history are key. Consider any underlying immune suppression - for example, renal dialysis patients have an associated immune suppression and may have needed to access healthcare facilities while abroad for their chronic health condition. Has the patient been visiting friends and relatives? These travellers may have a different risk profile from tourists who have only frequented the 'beaten track'.

\section{Destinations: the 'Where?'}

The destination information should be as granular as possible. It can be easier to think of it as zooming in from national to particular: Country $\rightarrow$ city/rural $\rightarrow$ local geography (mountains/lakes/beach) $\rightarrow$ accommodation (family/hostel/hotel). This should be completed for any country travelled through, and for the last 12 months. Review the resources for outbreaks (Table 1 ) to ensure specifically asking about travel to areas with malaria transmission and continuing outbreaks.

\section{Exposures: the 'What?'}

What was the patient doing while abroad? Again, record as much detail as possible:

- work: what setting?

- leisure: what activities?

- sexual contact: what and with whom?

- hospital treatment or admission?

- insect bites?

Table 2 outlines some specific risks and disease associations.

\section{Timeline: the 'When?'}

Review the 12 months leading up to this presentation. Be sure to establish the onset and progress of the symptoms. You will then be able to allocate diagnoses as 'in' or 'out' based on incubation 


\section{Resources for outbreaks}

- Travel Health Pro (formerly the National Travel Health Network and Centre (NathNaC)): Travel health information is produced by the NathNaC, a UK government organization. It is aimed at healthcare professionals advising travellers. Information is available by country, and as outbreak surveillance information. https://travelhealthpro.org.uk/

- ProMED: The Program for Monitoring Emergency Diseases (ProMED) is a programme of the International Society for Infectious Diseases. It is the largest publicly available system conducting global reporting of infectious disease outbreaks for up-to-the minute information on emerging and continuing outbreaks. https://promedmail.org/

- Imported Fever Service (IFS): This is a clinical advisory and specialist diagnostic service for medical professionals managing travellers who have returned to the UK with fever. After discussion with local microbiology, virology or infectious disease specialists, IFS can be consulted for advice and diagnostic panels to help secure a diagnosis. https://www.gov.uk/guidance/imported-fever-service-ifs

\section{Table 1}

periods It can be useful to think of events before, during and after each trip.

Before - are childhood vaccinations up to date? Has vaccination for country risk been completed before travel? For example, hepatitis A vaccination needs to be completed 14 days before travel. Did the individual take malaria prophylaxis? Were there travel medicine appointments?

During - dates of travel and exposures are key. Also establish a timeline for the onset of symptoms and signs. If the person was

\section{Activities and disease risks}

\section{Exposure}

Bites

Mosquito

Tick

Flea

Tsetse fly

Animal

\section{Activity}

Cruise ships

Caving

Farms

Fresh-water exposure

Game parks

Food/drink

Faecally contaminated water

Unpasteurized milk

Undercooked food

People

Sexual contact

Funerals

\section{Common}

Dengue, malaria

Lyme disease, tick typhus

\section{Occasional}

Chikungunya, West Nile fever, yellow fever, Japanese encephalitis, Zika virus

Q fever

Murine (endemic) typhus

Trypanosomiasis

Q fever, tularaemia

\section{Rare but important}

Filariasis

Other borreliosis (tick bite fever, relapsing fever), Crimean-Congo Haemorrhagic fever, ehrlichiosis, tick-borne encephalitis, tularaemia Plague

Anthrax, rabies, rat bite fever

Rabies

Acanthamoeba, melioidosis

Katayama fever (acute schistosomiasis), leptospirosis

Tick typhus

Anthrax, trypanosomiasis

Poliomyelitis

hepatitis $A / E$

Listeria, Salmonella, Shigella

Bacterial gastroenteritis, amoebiasis

Brucella

Trichinosis

Syphilis
HIV, hepatitis $A / B / C$, pelvic inflammatory disease

\section{Ebola}

Adapted from Johnston et al. Fever in returned travellers presenting in the United Kingdom: recommendations for investigations and initial management. Journal of Infection. 2009, 59:1-18 https://www.journalofinfection.com/article/S0163-4453(0900154-6/fulltext 


\section{Clinical syndromes and differential diagnoses}

\section{Fever and syndrome}

Incubation < 14 days

Undifferentiated fever

- Malaria (Plasmodium species)

- Dengue fever

- Campylobacteriosis, salmonellosis, shigellosis

- Typhoid fever (Salmonella enterica serotype Typhi)

- Acute human immunodeficiency virus infection

Fever with haemorrhage

- Viral haemorrhagic fever

Fever with respiratory findings

- Influenza

- Legionellosis (Legionella pneumophila)

- Q fever (Coxiella burnetii)

- SARS-1, MERS, SARS-CoV-2

\section{Incubation 14 days to 6 weeks}

Malaria

Typhoid fever

Hepatitis A

Hepatitis E

Acute schistosomiasis (Katayama fever)

Amoebic liver abscess (Entamoeba histolytica)

Incubation $>6$ weeks

Tuberculosis

Hepatitis B

Visceral leishmaniasis (L. donovani, $L$. chagasi, others)
Usual incubation period (range)

\section{Key investigations}

6 days to years

4-8 days (3-14 days)

$2-6$ days ( $1-20$ days)

7-18 days (3-60 days)

Acute illness, $10-28$ days (10 days -6 weeks)

3-14 days (usually $<21$ days)

$1-3$ days

5-6 days (2-10 days)

$14-21$ days ( $2-29$ days)

2-10 days (WHO and PHE definition

$<10$ days, SARS-CoV-2 up to 14 days)

6 days to years

$7-18$ days (3-60 days)

$28-30$ days (15-50 days)

26-42 days (2-9 weeks)

Katayama fever 4-8 weeks

Weeks to months

Primary, weeks; reactivation, years

60-90 days (45-180 days; rarely 9 months)

2-6 months (10 days to years)
Thick and thin blood films

Flavirus serology

MCS of faeces

Blood culture, stool culture

HIV test

\author{
Respiratory viral swab \\ Urinary antigen \\ Coxiella serology \\ Respiratory viral swab
}

As above

As above

HAV IgG/IgM

HEV IgG/IgM

Stool/urine OCP, serology

Liver ultrasound, serology

Sputum/tissue sample for AFB microscopy and culture

HBV serology

Histopathology (bone marrow/spleen)

AFB, acid-fast bacillus; HAV/HBV/HEV, hepatitis A/B/E virus; MCS, microscopy, culture and sensitivity; MERS, Middle East respiratory syndrome; OCP, ova, cysts and parasites; PHE, Public Health England; SARS, severe acute respiratory syndrome; SARS-CoV-2, severe acute respiratory syndrome coronavirus 2; WHO, World Health Organization.

Adapted from Incubation periods adapted from: Ryan et al. Illness after International Travel. NEJM 2002, 347 (7):505-516 https://www.nejm.org/doi/10.1056/ NEJMra020118

\section{Table 3}

supposed to be taking malaria prophylaxis, check their compliance.

After - when did they return to their home country? How have they been?

Table 3 shows the incubation periods for important imported diseases, ${ }^{4}$ which narrows down the possible diseases the patient could have, based on their timeline.

\section{Examination}

Use an 'A-E' (Airway, Breathing, Circulation, Disability, Exposure) approach to assess the stability of the patient before progressing to a top-to-toe examination. In addition to the cardiopulmonary, abdominal and neurology examination, a detailed skin examination is crucial. This may reveal rashes, bites, eschars, lymphadenopathy or even evidence of medical interventions. Remember to look under bra straps, in skin folds and on the feet.

\section{Investigations}

Having established a history and examined the patient, a basic investigation set should include a full blood count, renal function test, liver function tests, blood culture, blood smears and HIV test. The blood smear for malaria should be completed urgently - you should contact the duty biomedical scientist to inform them the sample is coming so they can ensure the result is available in a timely fashion, even if you are seeing the patient 
out-of-hours. The smear should be repeated three times in the first $24-72$ hours as sequestration of parasitized red cells means that malarial parasites are not always seen on a peripheral smear. Further investigations should be guided by the clinical syndrome, exposures and countries travelled to, but sending a respiratory viral swab, and urine for dipstick and microscopy, culture and sensitivity, also requesting a chest X-ray, is a good start. Table 3 lists the key investigations for important diseases.

\section{Management}

Early priorities of management are patient stabilization and infection control considerations. Could the patient have a syndrome compatible with a high-consequence infectious disease? If so, isolate them and have an early discussion with the local infectious disease service. There may be an urgent need to seek advice from the national public health service. Further treatment is determined by the most likely diagnosis. If the patient requires hospital admission, swabbing for resistant organisms is recommended, especially if there has been medical treatment abroad, as these travellers are at greater risk of being colonized by resistant bacteria. ${ }^{5}$

\section{Case studies}

\section{Case 1}

A 26-year-old man returned from Zanzibar with a fever and a rapidly deteriorating conscious state. He had sustained several mosquito bites while on holiday. A blood film demonstrated Plasmodium falciparum parasites with a parasitaemia of $6 \%$. Computed tomography (CT) of his brain demonstrated diffuse cerebral swelling. Intravenous artesunate was started within 5 hours of his arrival in hospital. Despite treatment, a second CT scan demonstrated progression of swelling and evidence of critically elevated intracranial pressure, and he died 14 hours after admission. Before travelling he had visited a travel health clinic that had advised against malaria prophylaxis as it was the low season. The patient had a history of splenectomy, which had not been appreciated as a risk factor for severe malaria.

Learning point -patients' personal risk factors affect their susceptibility to malaria, particularly its severe manifestations. In addition, always do a malaria film.

\section{Case 2}

A 54-year-old woman returned from Dubai with fever, cough and shortness of breath. She had been admitted to hospital in Dubai 2 days earlier with a respiratory infection and an oxygen requirement, but had self-discharged against medical advice and flown to the UK. A chest X-ray demonstrated left lower zone consolidation. C-reactive protein measured $513 \mathrm{mg} /$ litre. She fulfilled the case definition for possible Middle Eastern respiratory syndrome virus and was therefore isolated in a negative-pressure side room. Public Health England was consulted. A urinary antigen test came back positive for Legionella pneumophila. She was commenced on treatment with levofloxacin and made a good recovery.

Learning point - isolation precautions can always be deescalated, and not all imported infections turn out to be tropical.

\section{KEY REFERENCES}

1 Office for National Statistics. International Passenger Survey. https://www.ons.gov.uk/surveys/informationforhousehold sandindividuals/householdandindividualsurveys/internationalpass engersurvey (accessed 24 Mar 2021).

2 Angelo KM, Kozarsky PE, Ryan ET, Chen LH, Sotir MJ. What proportion of international travellers acquire a travel-related illness? A review of the literature. J Trav Med 2017 Sep 1; 24: 10.1093/jtm/ tax046. https://pubmed.ncbi.nlm.nih.gov/28931136/ (accessed 16 March 2021)

3 Buss I, Genton B, D'Acremont V. Aetiology of fever in returning travellers and migrants: a systematic review and meta-analysis. J Trav Med 2020; 27: taaa207. https://pubmed.ncbi.nlm.nih.gov/ 33146395/ (accessed 2 March 2021)

4 Ryan ET, Wilson ME, Kain KC. Illness after international travel. N Engl J Med 2002; 347: 505-16.

5 Department of Health and Social Care. UK 5-year action plan for antimicrobial resistance 2019 to 2024. https://www.gov.uk/ government/publications/uk-5-year-action-plan-for-antimicrobialresistance-2019-to-2024 (accessed 24 Mar 2021). 